\title{
MAPS-based vertex detectors: operational experience in STAR and future applications
}

\author{
Giacomo Contin* \\ Lawrence Berkeley National Laboratory, 1 Cyclotron Road, Berkeley, CA 94720, US \\ now at University of Liverpool, Oxford Street, Liverpool L69 7ZE, UK \\ E-mail: giacomo.continaliverpool.ac.uk
}

The STAR PiXeL detector (HFT PXL) at RHIC is the first application of the thin Monolithic Active Pixel Sensors (MAPS) technology in a collider environment. It is based on $50 \mu \mathrm{m}$-thin MAPS sensors with a pitch of $20.7 \mu \mathrm{m}$. The sensor is read-out in rolling shutter mode in $185.6 \mu \mathrm{s}$. The $170 \mathrm{~mW} / \mathrm{cm}^{2}$ power dissipation allows for air cooling and contributes to reducing the global material budget to $0.4 \%$ radiation length on the innermost layer. This system took data in $\mathrm{Au}+\mathrm{Au}$ collisions, $\mathrm{p}+\mathrm{p}$ and $\mathrm{p}+\mathrm{Au}$ collisions at $\sqrt{s_{N N}}=200 \mathrm{GeV}$ at RHIC, during the period 2014-2016. Operational experience and lessons learned from the construction and the 3 years of data-taking will be presented in this paper. Detector performance and results from $2014 \mathrm{Au}+\mathrm{Au}$ data analysis, demonstrating the STAR capabilities of charm reconstruction, will be shown. Following this successful experience, the next-generation MAPS sensor, featuring an integration time shorter than $20 \mu$ s, will be used to upgrade the ALICE Inner Tracking System (ITS) at LHC and has been proposed for the vertex detector (MVTX) for sPHENIX, the future nuclear physics experiment for the study of the QGP planned for RHIC. A short outlook on these future applications will conclude the paper.

The 26th International Workshop on Vertex Detectors

10-15 September, 2017

Las Caldas, Asturias, Spain

${ }^{*}$ Speaker. 


\section{Introduction}

The Monolithic Active Pixel Sensor (MAPS) technology integrates both sensor and readout electronics in one silicon device [1], allowing for the construction of excellent position resolution and low material budget devices. In recent years, the MAPS technology achieved a radiation tolerance and integration time performance which are adequate to the current high-energy heavy-ion experiments.

Ultimate-2, a first-generation MAPS developed by the PICSEL group at Institut Pluridisciplinaire Hubert Curien (IPHC), Strasbourg, France, was used to equip the two innermost layers of the STAR Heavy Flavor Tracker (HFT), which operated from 2014 to 2016 in the moderate RHIC radiation environment, featuring $185.6 \mu$ s integration time. The STAR HFT demonstrated the suitability of the MAPS technology for vertex detectors at colliders. This proceeding briefly summarizes the STAR HFT PiXeL (PXL) system in Section 2, describing the detector design and construction (2.1), reporting on the most important lessons learned from the three-years of operations (2.2), and presenting measured performance and physics achievements (2.3).

The next-generation MAPS sensor, named ALPIDE, will be used for the ALICE Inner Tracking System (ITS) Upgrade at LHC and has been proposed for the MAPS-based VerTeX detector (MVTX) of sPHENIX at RHIC. Capable to tolerate some $10^{13} 1 \mathrm{MeV} \mathrm{n}_{e q} / \mathrm{cm}^{2}$ and featuring a time resolution equal to $2 \mu \mathrm{s}$, ALPIDE will operate in the harsher radiation environment and high luminosity foreseen for these experiments. Highlights from the design, the expected performance and the project timeline of two of the future MAPS-based detector, the ALICE ITS, currently under construction, and the sPHENIX MVTX, recently proposed, are briefly discussed in Section 3.

\section{The first MAPS-based vertex detector: the STAR HFT PXL}

The HFT has been designed to improve the track pointing resolution of the existing STAR apparatus and to enable the direct topological reconstruction of short-lived charm hadron decays in the heavy-ion collision environment [2,3]. Heavy quark measurements are a key component for the systematic characterization of the dense medium created in heavy ion collisions, the so-called Quark-Gluon Plasma (QGP), which is one of the main goals of the STAR experiment heavy-ion program. This required resolution is achieved by tracking inwards from the TPC, which provides a pointing resolution of approximately $1 \mathrm{~mm}$, through a Silicon Strip Detector (SSD) layer and a silicon pad-based Intermediate Silicon Tracker (IST) layer, with pointing resolutions of 250$300 \mu \mathrm{m}$, to the PiXeL (PXL) detector. The two MAPS layers can point at secondary vertices with the resolution of a few tens of micrometers.

\subsection{Design}

The excellent track pointing resolution of the HFT is driven by the unprecedented characteristics of the PXL detectors: the small pixel pitch $(20.7 \mu \mathrm{m})$ featured by the sensor, the low contribution to the material budget of the inner layer, the position of the two cylindrical PXL layers (2.8 and $8 \mathrm{~cm}$ radii) with respect to the beam, and the pixel positional stability preserved at the level of $20 \mu \mathrm{m}$ by the mechanics. 
The cantilevered mechanics support on one side the two detector-halves. It allowed for fast insertion and retraction of the detector by manually sliding the detector-halves along rails inside a support cylinder and locking them into a reproducible position using kinematic mounts. Each half consists of 5 sectors mounted in dovetail slots. A sector represents the basic unit in terms of powering and readout and consists of a $250 \mu \mathrm{m}$ carbon fiber sector tube with four 10-sensor ladders mounted on each tube, one at the inner radius, and three at the outer radius, arranged in a turbo geometry design. A detector-half is shown in Figure 1.

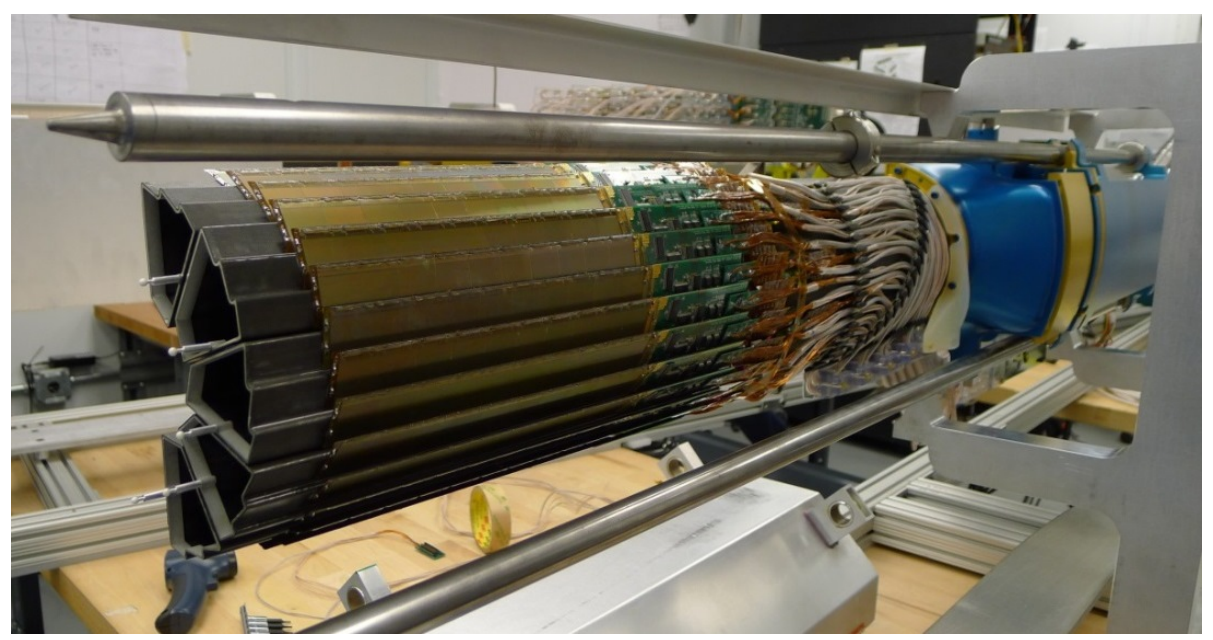

Figure 1: : PXL half-detector: 5 sectors mounted in dovetail slots on the carbon fiber support and connected to the insertion mechanics.

Ultimate-2 (see Figure 2) is the third MIMOSA-family sensor revision specifically developed for the STAR experiment $[4,5]$. This sensor features a high-resistivity epitaxial layer to increase the radiation hardness (measured up to $90 \mathrm{kRad} /$ year and $2 \cdot 10^{11}$ to $10^{12} 1 \mathrm{MeV} \mathrm{n} \mathrm{n}_{e q} / \mathrm{cm}^{2}$ ) and improve the signal-to-noise performance. The total signal for a MIP has been measured to be $\sim 1000 \mathrm{e}^{-}$, with a signal-to-noise ratio of about 30 for the pixel collecting most of the charge in the cluster. The pixel array is read out in a rolling-shutter fashion, by addressing one row at a time and processing all columns in parallel through programmable threshold discriminators located at the end of each column. The integration time of the whole sensor is $185.6 \mu \mathrm{s}$. A low power dissipation of $150 \mathrm{~mW} / \mathrm{cm}^{2}$ allows these sensors to be operated at room temperature with just air cooling.

This characteristic and the possibility to thin down the sensor to $50 \mu \mathrm{m}$, together with the use of aluminum conductor flexible cables and thin carbon fiber strucures, contributed to reducing the material budget on the innermost layer to less than $0.39 \%$ radiation lenght $X / X_{0}$.

\subsection{Operations experience}

The time taken from the beginning of the sensor development phase in 2003 to the initial data taking with a prototype detector was 10 years. A PXL detector prototype was installed in STAR in May 2013 and was operated in the Engineering Run for a month, with the goal of validating the design and the production processes. The PXL detector production began in summer 2013.

The PXL detector was finally installed and operated for the first time in STAR in January 2014. After being successfully commissioned, it collected $\sim 1.2$ Billion minimum-bias Au+Au events at 

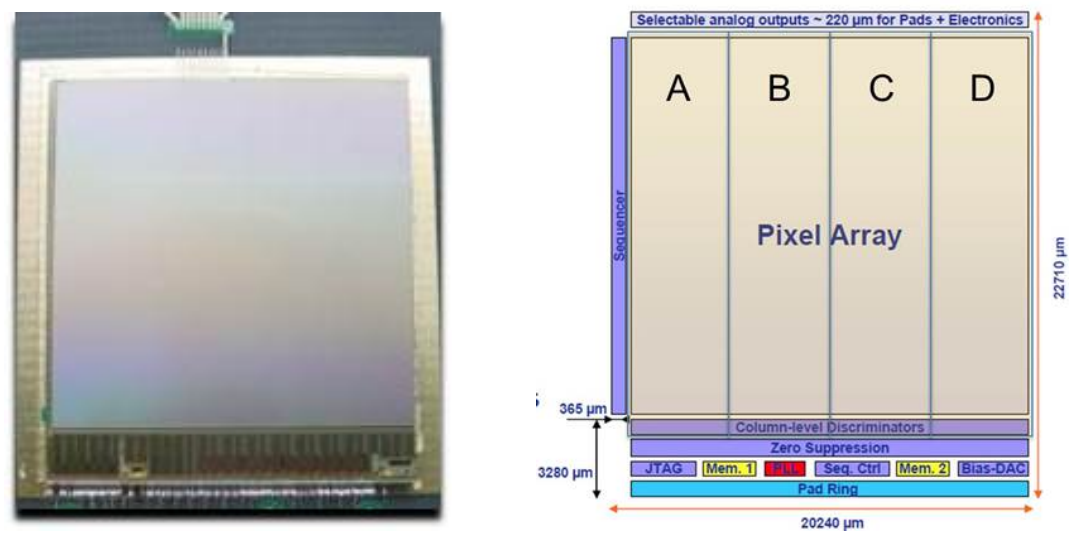

Figure 2: Left: Picture of an Ultimate-2 sensor wire-bonded to a testing board. Right: Block-diagram of the Ultimate-2 sensor.

$\sqrt{s_{N N}}=200 \mathrm{GeV}$ during the 2014 Run. In the 2015 Run it collected $\sim 1$ Billion $\mathrm{p}+\mathrm{p}$ and $\sim 0.6$ Billion $\mathrm{p}+\mathrm{Au}$ events. The data sample was completed in the $2016 \mathrm{Run}$, with $\sim 2$ Billion $\mathrm{Au}+\mathrm{Au}$ and $\sim 0.3$ Billion $\mathrm{d}+\mathrm{Au}$ events.

At the beginning of each Run, a PXL detector copy featuring an active channel fraction larger than $99 \%$ was installed in STAR. The onset of a latch-up induced damage decreased this fraction during the data taking period, as described in the following paragraphs. In the final year of operations, after constant improvements of the RHIC and STAR performance, the PXL detector took data at a typical trigger rate of $0.8-1 \mathrm{kHz}$ with a dead time of $\sim 6 \%$, handling a maximum number of pixels above threshold per sensor per event equal to 1000 (100) on the inner (outer) layer, corresponding to a maximum occupancy of $\sim 0.1 \%(\sim 0.01 \%)$. During data taking operations, each sector was reconfigured after a latch-up event at an average rate of $0.2 / \mathrm{min}$.

Many design and operational aspects were validated during the PXL detector operations over the experimental running periods [6]. For what concerns the mechanics, the detector halves maintained the surveyed pixel positions after insertion, during operational heating and in the cooling airflow of $10 \mathrm{~m} / \mathrm{s}$. The rapid insertion and removal mechanism using kinematic mounts allowed the removal and replacement of a complete operational second detector copy within 24 hours. The air cooling system maintained the maximum temperature on a sensor at $36.1{ }^{\circ} \mathrm{C}$ giving a maximum temperature rise of $13^{\circ} \mathrm{C}$ above the cooling airflow. In these cooling conditions, the temperature variation within a sensor was about $6^{\circ} \mathrm{C}$, with the maximum temperature measured in the digital periphery. The typical variation in sensor temperature over the runs was within $1-2^{\circ} \mathrm{C}$. From the sensor operations point of view, the current monitoring and latch-up protection circuitry limited the LU damage to a small number of sensors in a run after initial parameter tuning. The automated Single Event Upset (SEU) sensor reset and reconfiguration scheme on a 15 minute cycle limited SEU data corruption.

The major operational issues encountered by the PXL detector during the three years of physics data taking have been caused by the sensor sensitivity to latch-up event induced damage, and by the misbehavior of the readout firmware version in use for most of the 2015 Run. The latch-up induced damage manifested itself in different forms: increased digital current consumption, damage to pixel columns, loss of full or partial pixel sub-arrays. The failures occurred in the inner layer sensors $\sim 4$ 
times more frequently than on the outer layer sensors.

In the 2014 Run the damage was limited by reducing the over-current protection threshold from the default value ( $400 \mathrm{~mA}$ ) to $120 \mathrm{~mA}$ above the operating current. The latch-up protection circuit was optimized after the end of the 2014 operations. In the 2015 and 2016 RHIC Runs an over-current protection threshold at $80 \mathrm{~mA}$ above the operating current ( $\sim 1 \mathrm{~A}$ for the digital power) was applied to the running detector and limited significantly the onset of latch-up induced damage to 5 inner layer sensors in each Run.

The failure mechanism has been extensively studied through in a test campaign carried out in Fall 2014 at the 88" Cyclotron BASE Facility at LBNL. Existing PXL ladders and sensors have been exposed to heavy-ion and proton beam irradiation to measure the latch-up event cross-section and to reproduce the damage observed in STAR. This test allowed the characterization of the damage mechanism and for the definition of a safe operation procedure to set up the power supply current threshold settings for the subsequent data taking periods. The sensors tested included $50 \mu \mathrm{m}$ and $700 \mu \mathrm{m}$ full thickness devices with both high and low resistivity epitaxial layers. Current limited latch-up states have been observed with a typical increase of $300 \mathrm{~mA}$ in the operating digital current. Damage similar to the one observed in the STAR environment, with permanent increase of operating digital current and sensor data corruption, has been reproduced only on thinned highresistivity sensors. A further analysis of the damaged sensors through infrared camera inspection,

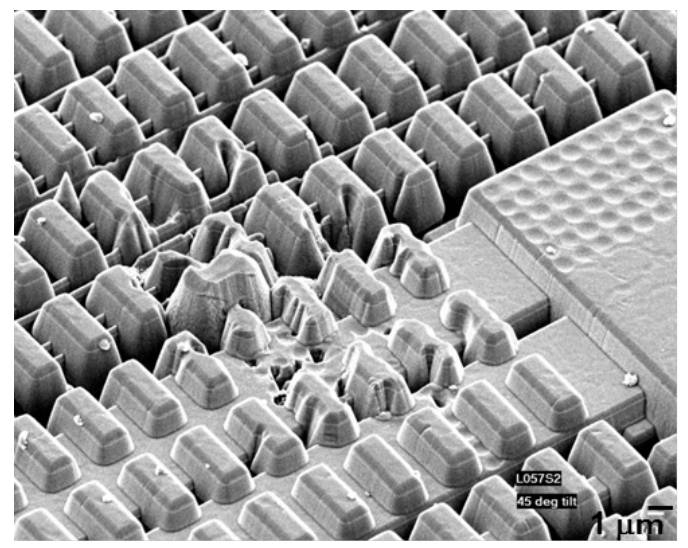

Figure 3: Scanning electronic microscope image of a damaged PXL sensor deconstructed through plasma etching technique (image taken at the Instrumentation Division laboratories - Brookhaven National Laboratory). The metal layer appears to be melted.

located the damage in specific structures of the digital section. The sensor substrate and sensitive epi-layer in the region of interest have been removed via plasma etching technique and examined through scanning electronic microscope at the BNL - Instrumentation Division laboratories (see Figure 3), showing a modification of the metal layer, which appears to be melted.

In the 2015 Run a flawed RDO firmware caused a partial PXL data loss and reduced the track matching efficiency to $\sim 40 \%$ on single HFT track reconstruction with respect to the efficiency measured in 2014. As demonstrated through a post-run investigation based on sensor illumination with an external LED, an unsafe implementation of a timing constraint caused the sensor frame reconstruction to fail: in each triggered PXL event, a fraction of reconstructed sensor data is not 
associated with the event itself. The extensive firmware tests carried out with pattern data and the performance of full detector calibrations before the firmware deployment were inadequate to spot this problem. The firmware was fixed and validated in 2016 and the new version was made available for future use.

\subsection{Performance and Physics}

The analysis of $\mathrm{Au}+\mathrm{Au}$ data collected in 2014 Run demonstrated that the track pointing resolution of the HFT system exceeded the design requirements, achieving $\sim 46 \mu \mathrm{m}$ for $750 \mathrm{MeV} / \mathrm{c}$ kaons for the 2 sectors equipped with aluminum cables on the inner layer, and better than $30 \mu \mathrm{m}$ for particle momenta $\geq 1 \mathrm{GeV} / \mathrm{c}$. The track pointing resolution in the azimuthal direction as a function of the particle momentum, as measured for the overall detector, is shown in Figure 4, left panel. This performance enabled the study of the $D$-meson production with a high significance signal. The measurement of the $D^{0} \rightarrow K \pi$ production in $\sqrt{s_{N N}}=200 \mathrm{GeV} \mathrm{Au}+\mathrm{Au}$ collisions for $1<\mathrm{p}_{T}<1.5 \mathrm{GeV} / \mathrm{c}$ (b) and $5<\mathrm{p}_{T}<10 \mathrm{GeV} / \mathrm{c}$ (c) is shown in Figure 4, right panel. The projected significance for the entire 2014 Run data sample reaches 220. The first measurement of the elliptic anisotropy $(v 2)$ of the charm meson $\mathrm{D}^{0}$ at midrapidity $(|\eta| \leq 1)$ in $\mathrm{Au}+\mathrm{Au}$ collisions at $\sqrt{s_{N N}}=200 \mathrm{GeV}$ has been published [7].

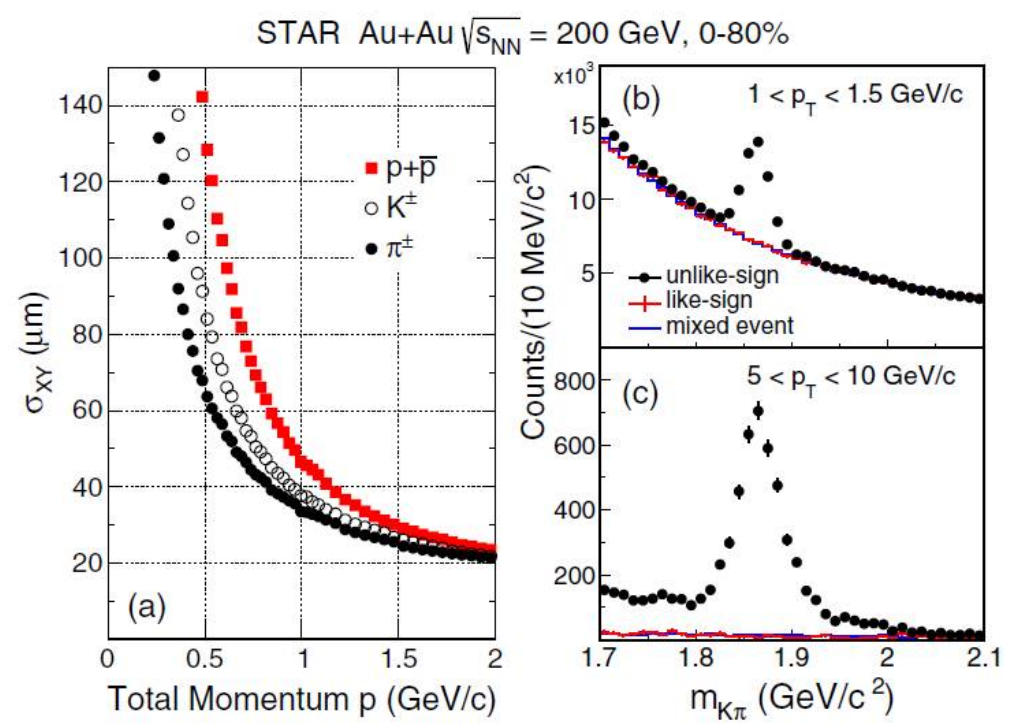

Figure 4: Left: Track pointing resolution in the azimuthal direction as a function of the particle momentum (a), measured for the overall detector. Similar resolution has been measured for the track pointing resolution along the beam direction. Right: $D^{0} \rightarrow K \pi$ production in $0 \%-80 \%$ centrality $\sqrt{s_{N N}}=200 \mathrm{GeV} \mathrm{Au+Au}$ collisions, for $1<\mathrm{p}_{T}<1.5 \mathrm{GeV} / \mathrm{c}$ (b) and $5<\mathrm{p}_{T}<10 \mathrm{GeV} / \mathrm{c}$ (c) [7].

Other measurements in the charm sector were performed using the HFT and are now being presented and published. The $\mathrm{D}_{s}$ production in Au+Au collisions at $\sqrt{s_{N N}}=200 \mathrm{GeV}$ has been measured [8], indicating an enhancement of $\mathrm{D}_{s}$ production in $\mathrm{Au}+\mathrm{Au}$ collisions with respect to $\mathrm{p}+\mathrm{p}$. The efficient suppression of the combinatorial background made possible by the HFT, enabled the first measurement of the $\Lambda_{c}$ production in heavy-ion collisions by the STAR experiment [9]. The 
measured $\Lambda_{c} / \mathrm{D}^{0}$ ratio shows a significant enhancement for $3<\mathrm{p}_{T}<6 \mathrm{GeV} / \mathrm{c}$ in $10-60 \%$ central $\mathrm{Au}+\mathrm{Au}$ collisions at $\sqrt{s_{N N}}=200 \mathrm{GeV}$ compared to the Montecarlo simulations, and is comparable to the measured baryon-to-meson ratios for light and strangeness hadrons.

The described performance demonstrated the success of the STAR HFT PXL project and validated the MAPS technology as suitable for vertex detector at colliders.

\section{The next-generation MAPS-based detectors}

The integration time featured by the STAR HFT Ultimate- 2 sensor, well suited for the charm meson decay vertex reconstruction, is not sufficiently short to allow the study of the rare bottom decays in the RHIC pile-up conditions. Compared to the STAR HFT, an integration time reduced by more than a factor of 10 is needed to allow high reconstruction efficiency, especially for low momentum tracks in high luminosity heavy-ion collisions, possibly maintaining an extremely lowmaterial budget on the innermost layer.

The next-generation MAPS sensor for the future high-energy heavy-ion experiment detectors, ALPIDE, improves the STAR HFT Ultimate-2 sensor in terms of radiation tolerance, limited power consumption and read-out time. Specifically designed for the ALICE ITS Upgrade detector, ALPIDE has been recently adopted also for the proposal of the MVTX, a new vertex detector for the future sPHENIX experiment at RHIC.

\subsection{The ALICE ITS Upgrade}

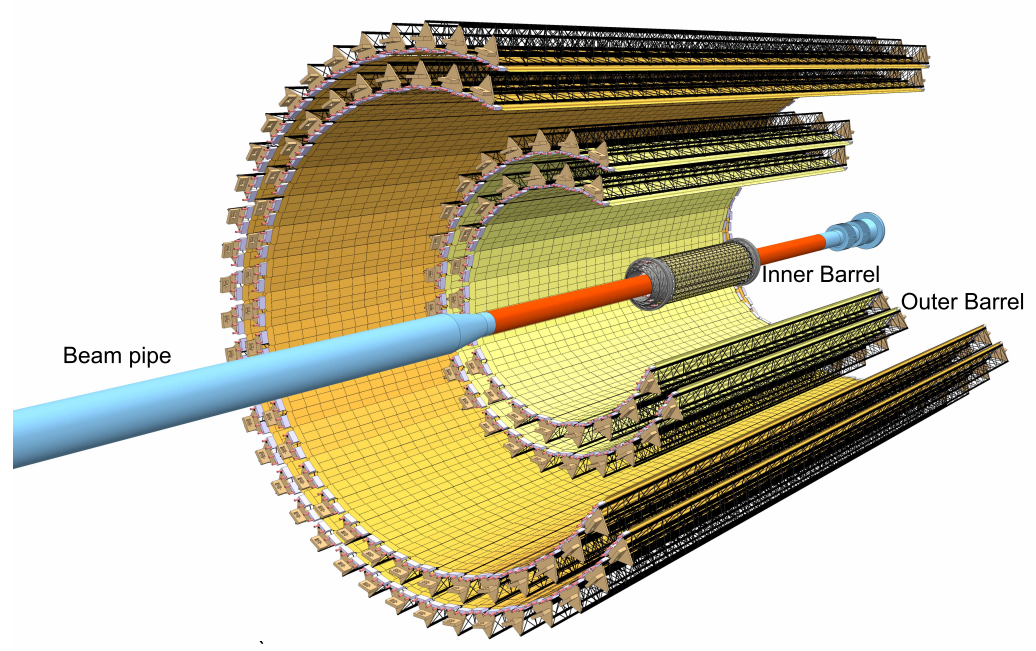

Figure 5: The general layout of the upgraded ITS detector.

The ALICE ITS Upgrade detector will consist of seven MAPS layers, with radii ranging from 22.4 to $400 \mathrm{~mm}$ from the interaction point [10]. The general layout of the upgraded ITS is shown in Figure 5. The material budget of the three innermost layers, as low as $0.3 \%$ of a radiation length, combined with the space-point precision of about $5 \mu \mathrm{m}$, will drive the expected track impact parameter resolution at $p_{T} \sim 500 \mathrm{MeV} / c$ to about $40 \mu \mathrm{m}$, which improves the current performance by a factor $\sim 3$ in the transverse plane and $\sim 6$ in the beam direction. The new detector will also 
allow for an efficient track reconstruction down to very low $\mathrm{p}_{T}$. The simulations show that the tracking efficiency at $\mathrm{p}_{T} \sim 0.1 \mathrm{GeV} / c$ will be improved by a factor $\sim 6$.

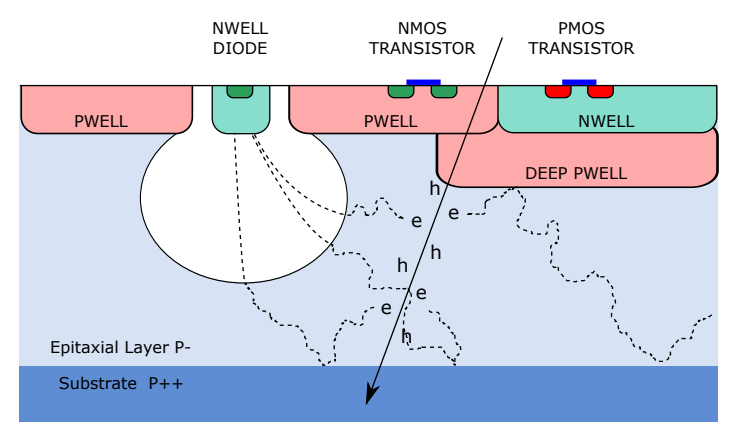

Figure 6: Schematic drawing of a monolithic pixel in the TowerJazz technology. The deep PWELL shields the PMOS transistor, allowing for full CMOS circuitry in the active area of the chip. A moderate negative voltage can be applied to the substrate to increase the depletion zone around the collection diode.

The ALPIDE sensor is produced in the TowerJazz $0.18 \mu \mathrm{m}$ CMOS imaging process [11]. It offers a deep PWELL, which can be used to shield the NWELL of PMOS transistors, as illustrated in Figure 6. This allows the use of full CMOS circuitry in the pixel area without the drawback of parasitic charge collection by the NWELLs. The process allows the use of a high-resistive epitaxial layer on a p-substrate, which increases the radiation tolerance. In addition, applying a moderate negative voltage to the substrate can be used to increase the depletion zone around the collection diode and improve the signal-to-noise ratio. The main characteristics of the sensor are:

- pixel size: $29 \times 27 \mu \mathrm{m}^{2}$,

- time resolution: about $2 \mu \mathrm{s}$,

- in-pixel discriminators and in-matrix address encoder with asynchronous sparsified readout,

- power consumption: $40 \mathrm{~mW} / \mathrm{cm}^{2}$,

- detection efficiency: $>99 \%$, with a accidental hit rate of $<10^{-6}$,

- high radiation tolerance: tested up to $2.7 \mathrm{Mrad}$ (TID), and $1.7 \times 10^{13} 1 \mathrm{MeV} \mathrm{n}_{\mathrm{eq}} / \mathrm{cm}^{2}$ (NIEL).

The upgraded ITS will be almost two orders of magnitude faster than the current detector. The read-out electronics will be able to record events at a typical rate of $50 \mathrm{kHz}$ and a few $100 \mathrm{kHz}$ for minimum bias $\mathrm{Pb}+\mathrm{Pb}$ and pp collisions respectively. The ITS Upgrade detector is expected to be operated after the end of the LHC Long Shutdown 2 (2021).

\subsection{The sPHENIX MVTX}

sPHENIX is the future nuclear physics experiment for multi-scaled studies of the QGP planned for RHIC. The baseline sPHENIX detector is optimized to employ light quark/gluon jets and Upsilons as probes that are sensitive to characteristic scales in the QGP, and does not include an inner vertex tracker. In order to expand the sPHENIX physics program to open-bottom physics measurements and other heavy-flavor observables, a MAPS-based silicon tracker for sPHENIX has been proposed. 
In order to deliver the desired physics goals with b-jets and B-mesons, requirements are placed on the detector design in the following aspects:

- Acceptance: the $b$-jet and $B$-meson physics programs are statistics-limited. Therefore it is important to provide a precision vertex displacement measurement for nearly all tracks detected by sPHENIX. The MVTX should provide full azimuthal coverage over the $|\eta|<1$ range for events within $|z|<10 \mathrm{~cm}$, matching the acceptance for the planned sPHENIX detector

- Event rate: in order to maximize the collected statistics, the inner tracker should deliver an event rate not lower than the sPHENIX trigger rate of $15 \mathrm{kHz}$.

- DCA resolution: the $c \tau$ for $D$ and $B$ decays is about $120 \mu \mathrm{m}$ and $460 \mu \mathrm{m}$, respectively. Therefore it is crucial to reach a DCA resolution $<50 \mu \mathrm{m}$ at $p_{T}>1 \mathrm{GeV} / c$ to distinguish tracks from heavy flavor hadron decays. This requires a material budget of few percent radiation lengths to minimize the multiple scattering contributions to the resolution.

- Efficiency: the $B$-meson physics program requires detection of both of the decay particle tracks from the $B \rightarrow D \rightarrow \pi^{ \pm} K^{\mp}$ decay chain. Therefore, a minimal efficiency $60 \%$ at $p_{T}=1$ $\mathrm{GeV} / c$ in central $\mathrm{Au}+\mathrm{Au}$ collisions is required.

The ALPIDE sensor developed for the ALICE ITS Upgrade has characteristics that meet the sPHENIX requirements. The MVTX design adapts the inner three layers of the ITS Upgrade (Inner Barrel) to the sPHENIX constraints and needs, leveraging the extensive R\&D work already done for ALICE on the following aspects:

- sensor design and production,

- Inner Barrel and mechanics layout,

- stave production and test,

- power system design,

- readout units design.

In addition to this, a specific R\&D and production effort will be focused on:

- data aggregation/formatting/DAQ interface development,

- Inner Barrel and mechanics layout,

- stave production and test,

- power system design,

- readout units design.

In the current plan, the sPHENIX MVTX is expected to be installed and to start taking physics data in 2022. 


\section{Conclusions}

The STAR HFT PXL, the first MAPS-based detector at a collider experiment, successfully completed the 3-year physics program at RHIC. Its performance exceeded the design requirements and enabled STAR to access the heavy flavor domain and to study the charmed hadron production at RHIC. The assembly and operations of the engineering run prototype were crucial to deal with a number of unexpected issues and to implement solutions before the Physics Runs. The PXL experience also demonstrated how short-lived detector projects can suffer from the limited time available to tune the data quality assurance tools. The PXL detector proved the MAPS technology as a suitable technology for vertex detectors.

The next-generation MAPS sensors, featuring a time resolution two orders of magnitude shorter, will be used for the ALICE ITS Upgrade at LHC, currently under construction, and have been proposed for the future sPHENIX MVTX detector at RHIC. These detectors are expected to be operated and take physics data after 2021 .

\section{References}

[1] C. Hu-Guo et al., First reticule size MAPS with digital output and integrated zero suppression for the EUDET-JRA1 beam telescope, Nucl.Instrum.Meth. A623 (2010) 480-482.

[2] J. Adams et al., Experimental and theoretical challenges in the search for the quark-gluon plasma: The STAR Collaboration's critical assessment of the evidence from RHIC collisions, Nucl. Phys. A 757 (2005) 102-183.

[3] C. Chasman et al., A Heavy Flavor Tracker for STAR, LBNL/PUB-5509-2008 (2008).

[4] A. Dorokhov et al., High resistivity CMOS pixel sensors and their application to the STAR PXL detector, Nucl.Instrum.Meth. A650 (2011) 174-177.

[5] I. Valin et al., A reticle size CMOS pixel sensor dedicated to the STAR HFT, JINST 7 (2012) C01102.

[6] L. Greiner and the STAR Collaboration, STAR MAPS Vertex Detector operational experience, PoS Vertex 2016 (2017) 007.

[7] STAR Collaboration, Measurement of $D^{0}$ Azimuthal Anisotropy at Midrapidity in Au+Au Collisions at $\sqrt{s_{N N}}=200 \mathrm{GeV}$, Phys. Rev. Lett. 118, 212301, (2017)

[8] Z. Long and STAR collaboration (2017), $D_{s}^{ \pm}$meson production in Au+Au collisions at $\sqrt{s_{N N}}=200 \mathrm{GeV}$ in STAR, J. Phys.: Conf. Ser. 779012028

[9] G. Xie and the STAR Collaboration, for. (2017). $\Lambda_{c}$ Production in Au+Au Collisions at $\sqrt{s_{N N}}=200$ GeV measured by the STAR experiment. Nuclear Physics A. 967 10.1016/j.nuclphysa.2017.06.004

[10] I. Belikov and the ALICE Collaboration, Upgrade of the ALICE Inner Tracking System, EPJ Web Conf. 125 (2016) 01004

[11] M. Keil and the ALICE Collaboration, Upgrade of the ALICE Inner Tracking System, JINST 10 C03012 (2015)

[12] N. Wermes (for the ATLAS CMOS Collaboration), Depleted CMOS pixels for LHC proton-proton experiments, Nucl.Instrum.Meth. A824 (2016) 483-486 